\title{
Correlation between tumor markers and type 2 diabetes mellitus complications and their related influencing factors
}

\author{
Ruonan Cai ${ }^{1}$, Qianqian Kong ${ }^{2}$, Zhaoling Wang ${ }^{1}$, Zhaohua Gao $^{1}$, Yan Huo ${ }^{1}$ \\ ${ }^{1}$ Department of Endocrinology, Xuzhou First People's Hospital, The Affiliated Hospital of China University of Mining and Technology, Xuzhou, \\ China; ${ }^{2}$ Department of Clinical Laboratory, Xuzhou First People's Hospital, The Affiliated Hospital of China University of Mining and Technology, \\ Xuzhou, China \\ Contributions: (I) Conception and design: R Cai; (II) Administrative support: Z Wang; (III) Provision of study materials or patients: Y Huo; (IV) \\ Collection and assembly of data: Q Kong; (V) Data analysis and interpretation: Z Gao; (VI) Manuscript writing: All authors; (VII) Final approval of \\ manuscript: All authors. \\ Correspondence to: Yan Huo. Department of Endocrinology, Xuzhou First People's Hospital, The Affiliated Hospital of China University of Mining \\ and Technology, Xuzhou, China. Email: huoyan7209@163.com.
}

Background: This study aims to investigate the correlation between type 2 diabetes mellitus (T2DM) and the tumor markers/biochemical parameters of patients, as well as the related factors leading to complications.

Methods: A total of 150 T2DM patients in our hospital were included as the research group, and 80 healthy persons were matched in the normal control group. The levels of tumor markers (CA199, CEA, CA153, CA125, AFP) and body mass index (BMI), waist-to-hip ratio (WHR), blood pressure (BP), fasting plasma glucose (FPG), glycosylated hemoglobin (HbA1c), urine microalbumin (M-ALB), and triglyceride (TG) in the two groups were determined. Based on the complications status of T2DM patients, the patients were further divided into a complication-free group (patients with simple diabetes) and complication group. Univariate analysis was performed between patients with and without complications.

Results: The levels of serum CA199, CEA, and CA125 in the study group were significantly higher than those in the normal control group (all $\mathrm{P}<0.0001$ ), and the levels of BMI, WHR, systolic BP (SBP), diastolic BP (DBP), FPG, HbA1c, M-ALB, and TG in the study group were significantly higher than those in the control group (all $\mathrm{P}<0.05$ ). Tumor markers CA199, CEA, and CA125 were positively correlated with BMI, WHR, BP, FPG, HbA1c, M-ALB, and TG. Smoking, family history of diabetes, combined hypertension, hyperlipemia, course of disease, CA199, CEA, CA153, CA125, AFP, SBP, DBP, FPG, HbA1c, M-ALB, and TG were the influencing factors of complications in T2DM patients.

Conclusions: Relevant indicators of T2DM patients with complications should be fully evaluated clinically, and long-term follow-up observation should be conducted, so as to reduce the occurrence of complications.

Keywords: Type 2 diabetes mellitus (T2DM); complication; tumor markers; influencing factor

Submitted Oct 21, 2021. Accepted for publication Jan 12, 2022.

doi: 10.21037/apm-21-3429

View this article at: https://dx.doi.org/10.21037/apm-21-3429

\section{Introduction}

In 2019, the International Diabetes Federation (IDF) released the 9th edition of the IDF Diabetes Atlas, which shows that the prevalence of diabetes in adults $(20$ 79 years old) worldwide is about $9.3 \%$ (1). In China, the case fatality rate of diabetes is also on the rise. A study (2) showed that compared to 2010 , the total standardized mortality rate of diabetes in China in 2017 increased, with an average annual growth rate of $2.8 \%$. Type 2 diabetes mellitus (T2DM) is the main type of diabetes mellitus in China, and its prevalence increased from $2.5 \%$ 
in 1994 to $10.9 \%$ in 2013 (3). T2DM is caused by the relative deficiency of insulin and is closely related to the oxidative stress and inflammatory response of the body (4). Long-term hyperglycemia in diabetic patients can lead to chronic damage and dysfunction in human tissues and organs such as the heart, liver, kidney, and blood vessels (5). A number of clinical studies have pointed out that diabetes is highly correlated with the occurrence and development of tumors (6). In 2010, the American Diabetes Association (ADA) and the American Cancer Society (ACS) jointly published a consensus stating that diabetes patients with T2DM are at a higher risk of cancer, including endometrial, pancreatic, colon, bladder, and breast cancers. Liu et al. (7) showed that the risk of endometrial cancer increases 2-fold, pancreatic cancer 1.8-fold, colorectal cancer 1.3-fold, and breast cancer slightly in T2DM patients.

It has now become the norm to perform malignant tumor screening in diabetic patients in the clinic. Tumor markers are biological substances that are spontaneously synthesized and secreted by tumor cells during the occurrence and development of malignant tumors or that obviously respond to tumor cells and indicate tumor presence. They can be detected in human blood, body fluids, cells, and tissues, and can be used in the clinical diagnosis of tumors and the evaluation of prognosis (8). A previous study found that T2DM patients have a higher risk of developing malignant tumors than healthy people (9). Therefore, tumor marker screening in T2DM patients has certain clinical significance. Understanding the major risk factors of T2DM complications and their correlation with tumor markers can better control the development of diabetes, extend the life span of patients, reduce the mortality, and improve the quality of life of patients. Based on this, 150 T2DM patients were included as research subjects in this study, aiming to investigate the relationship between T2DM patients and tumor markers/biochemical indicators, as well as the risk factors leading to complications, so as to aid in the prevention and treatment of diabetes complications in clinical practice. We present the following article in accordance with the STROBE reporting checklist (available at https://apm.amegroups.com/article/view/10.21037/apm$21-3429 / \mathrm{rc})$.

\section{Methods}

\section{Clinical data of subjects}

A total of $150 \mathrm{~T} 2 \mathrm{DM}$ patients in our hospital were enrolled as the study group, and 80 healthy persons were matched in the normal control group. There were 80 males and 70 females in the study group, with an average age of $55.26 \pm 10.02$ years. There were 48 males and 32 females in the normal control group, with an average age of $52.89 \pm 9.68$ years. There was no statistically significant difference in the baseline data of gender, age, education level, and occupation between the two groups (all $\mathrm{P}>0.05$ ), indicating comparability (Table 1).

The inclusion criteria were as follows: (I) patients met the WHO criteria for the diagnosis of T2DM (10), including typical diabetic symptoms plus random blood glucose $\geq 11.1 \mathrm{mmol} / \mathrm{L}$, fasting blood glucose $(\mathrm{FBG}) \geq 7.0 \mathrm{mmol} / \mathrm{L}$, or blood glucose $\geq 11.1 \mathrm{mmol} / \mathrm{L}$ at $2 \mathrm{~h}$ after glucose loading; (II) complete clinical data.

The exclusion criteria were as follows: (I) the patients had diabetic ketoacidosis or non-ketotic hyperosmolar state; (II) the patients had serious abnormalities of heart, liver, and kidney function; (III) the patients were in the acute and chronic inflammatory period, and the body was in a state of stress; (IV) the patients were complicated with malignant or benign tumors, and the malignant or benign tumors were detected during follow-up; (V) the patients had mental disorders or long-term use of related drugs; (VI) the patients had secondary diabetes or gestational diabetes; (VII) the patients suffered from autoimmune diseases, or glucocorticoid use affected human glucose metabolism.

All procedures performed in this study involving human participants were in accordance with the Declaration of Helsinki (as revised in 2013). The study was approved by the ethics committee of The Affiliated Hospital of China University of Mining and Technology (No. LLSL-2021-62) and informed consent was taken from all the patients.

\section{Observation indexes}

Detection method of tumor markers: after fasting for 8-12 h, all patients and healthy controls underwent a health examination and venous blood was obtained on an empty stomach the next morning. The samples were centrifuged to obtain serum for detection. The tumor markers CA199, CEA, CA153, CA125, and AFP were measured by the Swiss Roche automatic biochemical immunoanalyzer and electrochemiluminescence method.

Detection methods of body mass index (BMI), waistto-hip ratio (WHR), blood pressure (BP), fasting plasma glucose (FPG), glycosylated hemoglobin (HbA1c), urine 
Table 1 Comparison of clinical data between the two groups

\begin{tabular}{|c|c|c|c|c|}
\hline Feature & Study group $(n=150)$ & Control group $(n=80)$ & $\chi^{2} / t$ & $\mathrm{P}$ \\
\hline Gender, n (\%) & & & 0.940 & 0.332 \\
\hline Male & $80(53.3)$ & $48(60.0)$ & & \\
\hline Female & $70(46.7)$ & $32(40.0)$ & & \\
\hline Junior middle school and below & $42(28.0)$ & $21(26.3)$ & & \\
\hline Senior middle school & $57(38.0)$ & $32(40.0)$ & & \\
\hline Junior college or above & $51(34.0)$ & $27(33.7)$ & & \\
\hline Occupation, n (\%) & & & 0.047 & 0.997 \\
\hline Farmer & $48(32.0)$ & $25(31.3)$ & & \\
\hline Other & $35(23.3)$ & $19(23.8)$ & & \\
\hline
\end{tabular}

microalbumin (M-ALB), and triglyceride (TG): the height and weight of subjects were recorded, and BMI was calculated based on the measurement results. Waist circumference and hip circumference were measured by soft tape, and WHR was calculated based on the results. BP was measured with a sphygmomanometer in the morning, and systolic BP (SBP) and diastolic BP (DBP) were recorded respectively. Fasting venous blood was obtained from the enrolled subjects, and serum was obtained after conventional centrifugation. HbA1c was detected by the Burrill HbA1c meter. FPG and TG were detected by the Siemens automatic biochemical analyzer. The urine of the patients was collected under the fasting state in the morning, and the level of M-ALB was analyzed by an automatic urine analyzer. All operations were carried out in strict accordance with the instructions of the kit and instrument.

\section{Research methods}

The two groups of subjects were observed and compared in terms of the levels of tumor markers CA199, CEA, CA153, CA125, and AFP and the parameters BMI, WHR, BP, FPG, HbA1c, M-ALB, and TG. The correlation between these two types of indexes was analyzed. The patients in the study group were divided into a complication group and complication-free group. Univariate analysis was performed to analyze the general clinical data of patients and the influencing factors leading to complications.

\section{Statistical analysis}

The data were sorted and statistically analyzed using SPSS 25.0 software. The inspection level was $\alpha=0.05$, and $\mathrm{P}<0.05$ was considered statistically significant. The count data were shown as frequency and percentage, and compared by the $\chi^{2}$ test of independent samples. Measurement data were shown as mean \pm standard deviation $(\bar{x} \pm s)$.

\section{Results}

\section{Comparison of tumor markers between the two groups}

The levels of serum CA199, CEA, and CA125 in the study group were significantly higher than those in the normal control group (all $\mathrm{P}<0.0001)$. The levels of serum CA153 and AFP in the study group were higher than those in the normal control group, but with no significant difference (both $\mathrm{P}>0.05$; Table 2, Figure 1).

\section{Comparison of BMI, WHR, BP, FPG, HbA1c, M-ALB, and TG between the two groups}

The levels of BMI, WHR, SBP, DBP, FPG, HbA1c, M-ALB, 
Table 2 Comparison of tumor marker levels between the two groups $(\bar{x} \pm s)$

\begin{tabular}{|c|c|c|c|c|c|}
\hline Group & CA199 (U/mL) & CEA (ng/mL) & CA153 (U/mL) & CA125 (U/mL) & $\operatorname{AFP}(\mathrm{ng} / \mathrm{mL})$ \\
\hline Control group $(n=80)$ & $11.16 \pm 2.05$ & $2.08 \pm 0.52$ & $10.28 \pm 1.48$ & $6.89 \pm 1.08$ & $2.86 \pm 0.86$ \\
\hline$t$ & 20.80 & 17.80 & 1.960 & 21.88 & 1.597 \\
\hline$P$ & $<0.0001$ & $<0.0001$ & 0.051 & $<0.0001$ & 0.112 \\
\hline
\end{tabular}

A

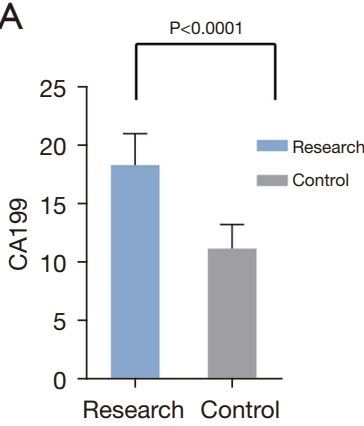

D

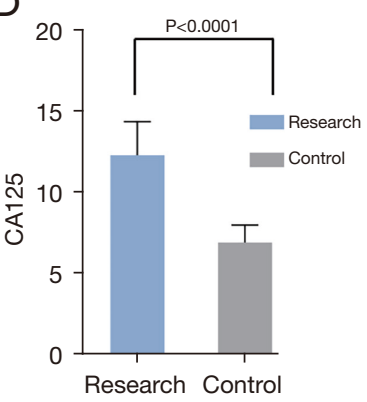

B

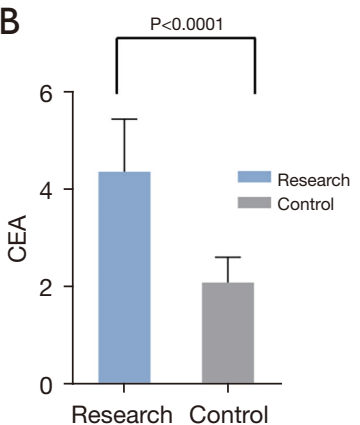

$E$

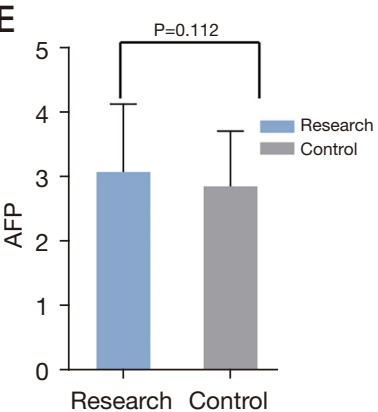

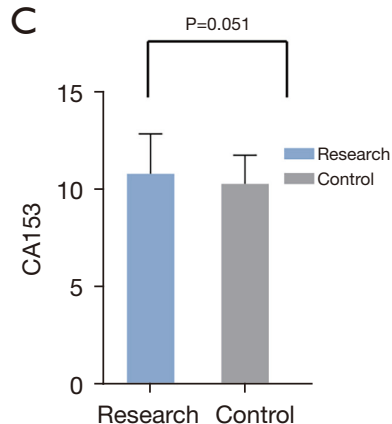

Figure 1 Histograms of tumor markers in the two groups. Comparison of CA199 (A), CEA (B), CA153 (C), CA125 (D) and AFP (E) levels.

and TG in the study group were significantly higher than those in the normal control group (all $\mathrm{P}<0.05$; Table 3, Figure 2).

\section{Correlation analysis between tumor markers and} parameters of BMI, WHR, BP, FPG, HbA1c, M-ALB, and $T G$

As shown in Table 4, Pearson correlation analysis results showed that tumor markers CA199, CEA, and CA125 were significantly positively correlated with BMI, WHR, BP, FPG, HbA1c, M-ALB, and TG.

\section{Univariate analysis of the clinical data of patients with and without complications}

Table 5 shows that smoking, family history of diabetes, hypertension, hyperlipidemia, course of disease, CA199, CEA, CA153, CA125, AFP, SBP, DBP, FPG, HbA1c, $M-A L B$, and TG were the influencing factors of complications in patients with T2DM.

\section{Discussion}

The incidence rates of T2DM and malignant tumors have been increasing in recent years. Gata et al. (11) pointed out that in both developing and developed countries, the mortality rates of T2DM and malignant tumors are among the highest, which has prompted many clinical researchers to pay attention to the relationship between the two. Many studies suggest that there is a significant correlation between T2DM and malignant tumors $(12,13)$. A study by Zhao et al. (12) shows that the incidence rates 
Table 3 Comparison of BMI, WHR, BP, FPG, HbA1c, M-ALB, and TG between the two groups ( $\bar{x} \pm$ s)

\begin{tabular}{|c|c|c|c|c|c|c|c|c|}
\hline Group & BMI $\left(\mathrm{kg} / \mathrm{m}^{2}\right)$ & WHR (value) & $\mathrm{SBP}(\mathrm{mmHg})$ & $\mathrm{DBP}(\mathrm{mmHg})$ & $\mathrm{FPG}(\mathrm{mmol} / \mathrm{L})$ & $\mathrm{HbA1c}(\%)$ & M-ALB $(\mu \mathrm{g} / \mathrm{min})$ & $\mathrm{TG}(\mathrm{mmol} / \mathrm{L})$ \\
\hline$t$ & 12.73 & 2.67 & 27.90 & 9.24 & 17.97 & 19.05 & 14.54 & 37.63 \\
\hline
\end{tabular}

BMI, body mass index; WHR, waist-to-hip ratio; BP, blood pressure; FPG, fasting plasma glucose; HbA1c, glycosylated hemoglobin; M-ALB, urine microalbumin; TG, triglyceride; SBP, systolic BP; DBP, diastolic BP.
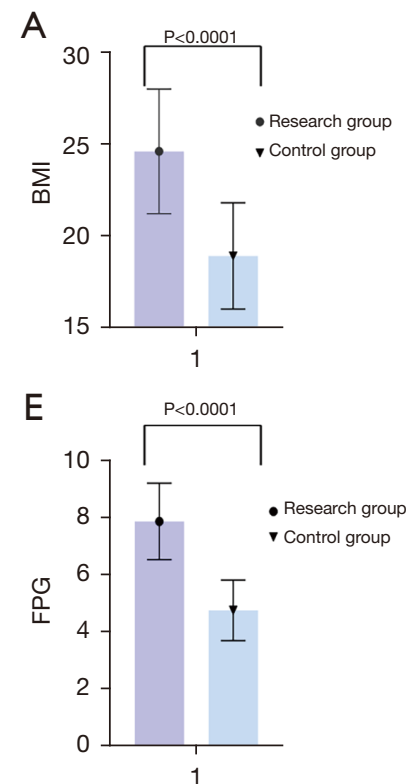

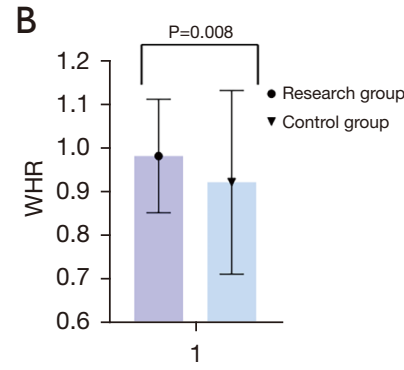

$\mathrm{F}$

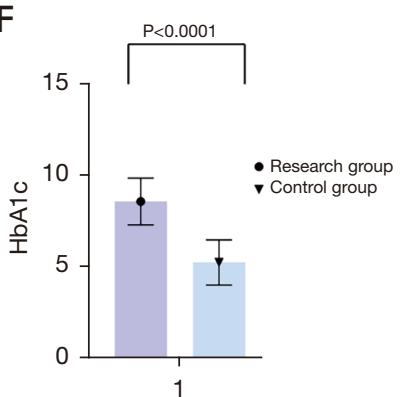

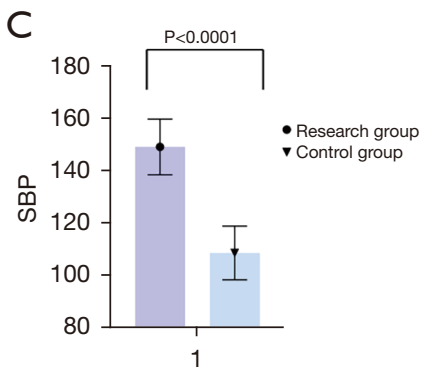

G

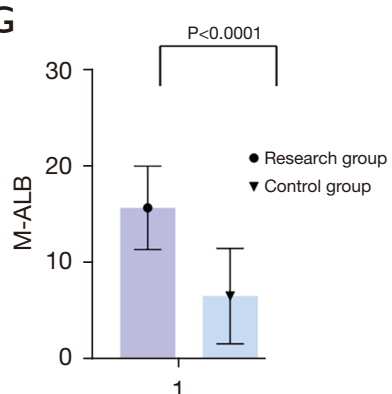

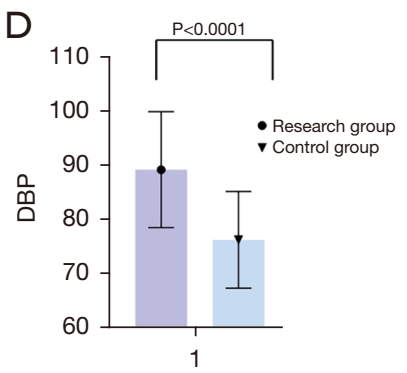

$\mathrm{H}$

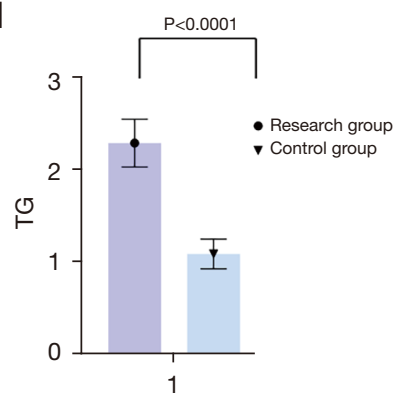

Figure 2 Comparison of BMI, WHR, BP, FPG, HbAlc, M-ALB, and TG between the two groups. Comparison of BMI (A), WHR (B), SBP (C), DBP (D), FPG (E), HbA1c (F), M-ALB (G), and TG (H) between the two groups. BMI, body mass index; WHR, waist-to-hip ratio; BP, blood pressure; FPG, fasting plasma glucose; HbA1c, glycosylated hemoglobin; M-ALB, urine microalbumin; TG, triglyceride; SBP, systolic BP; DBP, diastolic BP.

of liver cancer, pancreatic cancer, and endometrial cancer are the highest among diabetic patients, especially those with T2DM, followed by colorectal cancer, breast cancer, and bladder cancer. The mechanism of T2DM in inducing tumors is controversial in clinical practice. However, a study by Liu et al. indicates that the occurrence of malignancies is related to various factors, including blood glucose, insulin, insulin resistance, adipocytokines, and trace elements (14). Chronic complications of diabetes are common symptoms of diabetes patients with poor blood glucose control and late diabetes. The quality of life of patients with diabetes complications is significantly reduced. Therefore, investigating the relationship between tumor markers and complications in T2DM patients as well as the influencing factors of complications can aid in the treatment of patients in clinical practice.

Previous studies have shown that the type of disease and related complications in patients with $\mathrm{T} 2 \mathrm{DM}$ are more complex, and some complications can lead to elevated tumor markers (15). The present study also showed that the levels of serum CA199, CEA, and CA125 in the study group were significantly higher than those in the normal control group 
Table 4 Correlation analysis between tumor markers and parameters of BMI, WHR, BP, FPG, HbA1c, M-ALB, and TG ( $\mathrm{n}=230$ )

\begin{tabular}{|c|c|c|c|c|c|c|c|c|c|}
\hline Index & Test value & BMI & WHR & SBP & DBP & FPG & $\mathrm{HbA1c}$ & M-ALB & $\mathrm{TG}$ \\
\hline CA199 & Significance (two-tailed) & $<0.001$ & 0.001 & $<0.001$ & $<0.001$ & $<0.001$ & $<0.001$ & $<0.001$ & $<0.001$ \\
\hline \multirow[t]{2}{*}{ CEA } & Pearson correlation & $0.471^{\star \star}$ & $0.199^{\star \star}$ & $0.709^{\star *}$ & $0.447^{\star \star}$ & $0.610^{\star *}$ & $0.565^{\star \star}$ & $0.458^{\star \star}$ & $0.695^{\star \star}$ \\
\hline & Significance (two-tailed) & $<0.001$ & 0.002 & $<0.001$ & $<0.001$ & $<0.001$ & $<0.001$ & $<0.001$ & $<0.001$ \\
\hline CA153 & Significance (two-tailed) & 0.059 & 0.926 & 0.029 & 0.008 & 0.069 & 0.008 & 0.004 & 0.019 \\
\hline \multirow[t]{2}{*}{ CA125 } & Pearson correlation & $0.481^{\star \star}$ & $0.154^{*}$ & $0.708^{\star *}$ & $0.445^{\star \star}$ & $0.571^{\star *}$ & $0.606^{\star *}$ & $0.508^{\star *}$ & $0.714^{\star \star}$ \\
\hline & Significance (two-tailed) & $<0.001$ & 0.019 & $<0.001$ & $<0.001$ & $<0.001$ & $<0.001$ & $<0.001$ & $<0.001$ \\
\hline AFP & Pearson correlation & $0.146^{*}$ & -0.001 & 0.083 & -0.023 & 0.022 & 0.100 & 0.107 & 0.098 \\
\hline
\end{tabular}

* indicates significant, with $\mathrm{P}$ values between 0.01 and 0.05 ; ${ }^{* *}$ means extremely significant, $\mathrm{P}$ value below 0.01 . BMI, body mass index; WHR, waist-to-hip ratio; BP, blood pressure; FPG, fasting plasma glucose; HbA1c, glycosylated hemoglobin; M-ALB, urine microalbumin; TG, triglyceride; SBP, systolic BP; DBP, diastolic BP.

(all $\mathrm{P}<0.0001$ ). The occurrence of diabetes complications is mainly caused by poor control of related biochemical indicators in patients, and FPG, HbA1c, M-ALB, and TG are key indicators to determine the severity and prognosis of diabetes patients (16). This study also found that BMI, WHR, SBP, DBP, FPG, HbA1c, M-ALB, and TG levels in the study group were significantly higher than those in the normal control group (all $\mathrm{P}<0.05$ ). Pearson correlation analysis in this study showed that tumor markers CA199, CEA, and CA125 were significantly positively correlated with BMI, WHR, BP, FPG, HbA1c, M-ALB, and TG, which was basically consistent with the results of a number of previous studies $(17,18)$. Normal cells underwent malignant transformation under the action of stimulating factors and became malignant tumor cells with relatively strong glycolysis level. Especially when diabetic patients suffer from acute metabolic abnormalities, such as when ketoacidosis occurs, AFP, CEA, CA199 increase, which is closely related to the concentration of blood sugar. On the other hand, long-term uncontrolled blood glucose may lead to digestive tract damage due to the effect of "glycotoxicity", resulting in abnormally high results of CA199, CEA and CA125.

The present study shows that smoking, family history of diabetes, hypertension, hyperlipidemia, course of disease, CA199, CEA, CA153, CA125, AFP, SBP, DBP, FPG, HbA1c, M-ALB, and TG are the influencing factors of complications in T2DM patients. The complications of diabetes mainly include acute complications and chronic complications. The main acute complications were diabetic ketoacidosis, hyperglycemia, hyperosmolar state and hypoglycemia. The main cause of chronic complications is the damage of vascular endothelium caused by long-term hyperglycemia, which mainly includes microvascular disease and macrovascular disease. Microangiopathy is mainly diabetic retinopathy, diabetic nephropathy and diabetic peripheral neuropathy. Macrovascular diseases include serious cardiovascular and cerebrovascular events such as myocardial infarction, cerebrovascular disease and even stroke. The occurrence of T2DM complications is related to many factors, including genetics, age, gender, blood sugar control level, diabetes course, and other cardiovascular risk factors. Bai et al. (19) showed that the course of disease is the main factor leading to the complications of T2DM patients, so health education of Chinese residents should be strengthened to reduce the occurrence of complications and improve the quality of life of patients. T2DM is a low-grade chronic inflammatory disease, and the increased expression of IL-6 and TNF- $\alpha$ can lead to chronic pancreatic injury. Meanwhile, the increase of glycosylation end products and HbAlc in the state of hyperglycemia can render lipoproteins in the pancreatic arterial wall more susceptible to oxidative modification. This leads to vascular endothelial damage, which further aggravates the pancreatic injury, resulting in degeneration and even necrosis of pancreatic duct cells (20). CA199 is expressed by pancreatic exocrine department 
Table 5 Univariate analysis results of the clinical data of patients with and without complications

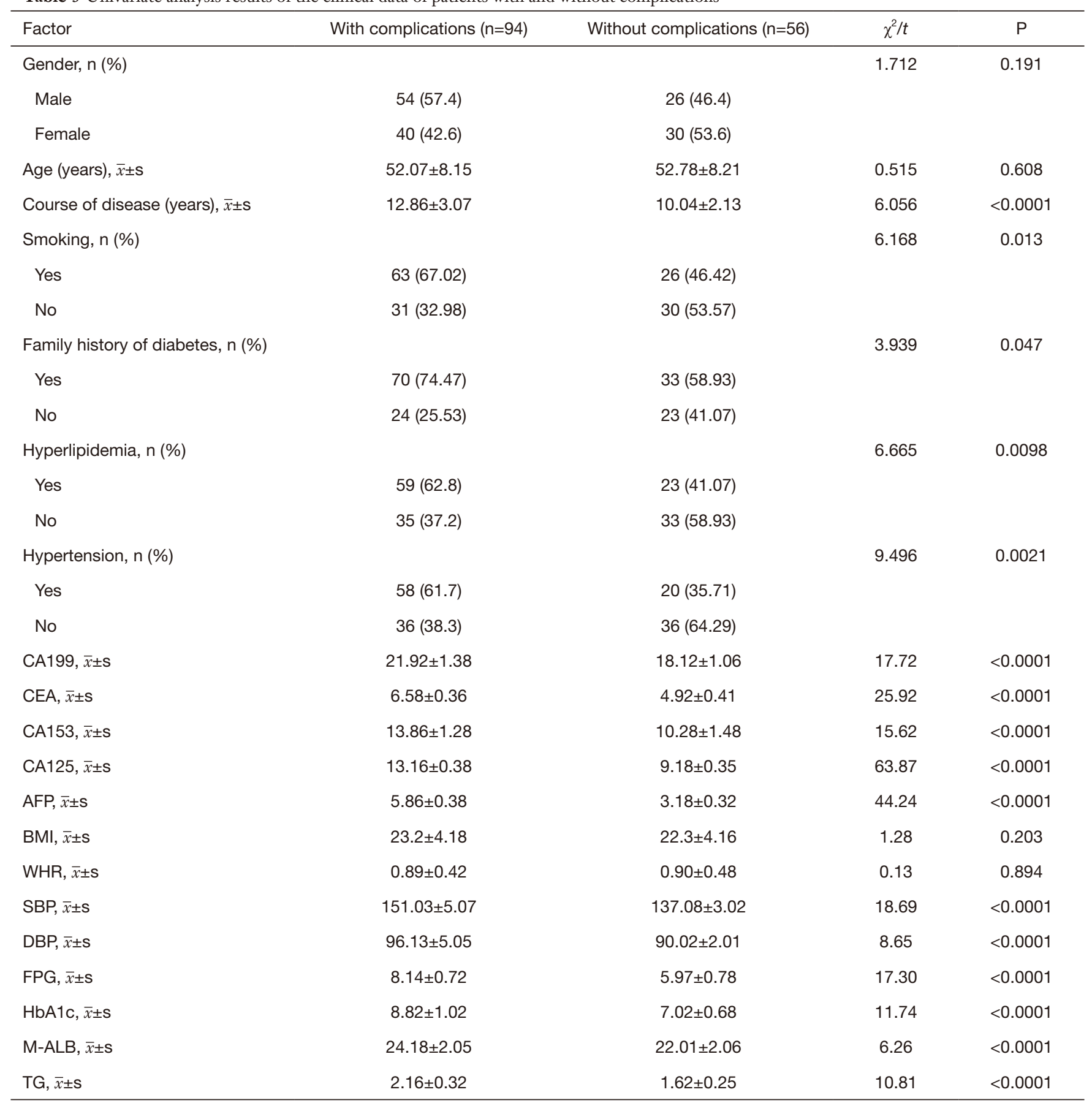

BMI, body mass index; WHR, waist-to-hip ratio; BP, blood pressure; SBP, systolic BP; DBP, diastolic BP; FPG, fasting plasma glucose; $\mathrm{HbA1c}$, glycosylated hemoglobin; M-ALB, urine microalbumin; TG, triglyceride.

and can be used as a sensitive indicator to screen for pancreatic exocrine injury. Histological changes of islets in T2DM patients are related to the inflammatory process of pancreatic exocrine department (21). In addition, mildly elevated blood lipase and amylase levels were associated with a weak hypodensity of the head of the pancreas in patients with hyperglycemia, leading to subclinical and mild forms of islet inflammation. This islet inflammation is caused by 
the activation of the innate immune system by metabolic stress and mediated by IL-1 signaling. A moderately elevated glucose concentration $(11 \mathrm{mmol} / \mathrm{L})$ was sufficient to induce transcriptional activation of IL-1 expression in the islets (22). Similarly, a previous study has shown that free fatty acids also promote inflammatory responses (23). Therefore, islet inflammation and destruction of islet cells caused by hyperglycemic toxicity may be the reason for elevated CA199 level.

The results of this study showed that CEA levels were significantly increased in patients with diabetes complications, and vascular endothelial growth factor levels were also increased in response to stress. There is a clear relationship between diabetic complications and endothelin, which also plays key roles in the mechanisms of tumors, such as participating in cell proliferation and the tumorization of abnormal proliferative tissues. CEA is a highly glycosylated cell surface glycoprotein, expressed on the surface of inflammatory cells as an adhesion molecule. Some studies have shown that CEA levels are elevated in inflammation-related diseases such as metabolic syndrome (24). In addition, a previous study reported an association between CEA levels and markers of oxidative stress (25). High blood sugar affects the formation of free radicals and may ultimately lead to increased oxidative stress. Severe oxidative stress and poor glycemic control may lead to increased CEA expression. Lu et al. (26) pointed out that the worse the blood glucose control, the higher the CA125 level, and the higher the CA125 level in patients with complications. Complications are important criteria which reflect the blood glucose control of patients, and the level of CA125 is positively correlated with blood glucose-related indexes. Increased AFP is related to pancreatic cancer, colorectal cancer, and liver cancer, and AFP is also an independent risk factor for T2DM complications. This is mainly due to the disorder of lipid metabolism in the human body, which improves the reproduction of tumor markers and indirectly promotes the occurrence and development of liver cancer, pancreatic cancer, and colorectal cancer. Xie et al. (27) showed that BP control also has an important relationship with blood glucose level, and people with a high blood glucose level also have relatively high BP. Therefore, the control of stable BP in T2DM patients is also a key way to reduce complications. Studies have shown that, diabetes can increase the risk of a variety of malignant tumors such as stomach cancer (28), bladder cancer (29), colorectal cancer (30), ovarian cancer (31), and pancreatic cancer (32).
The mechanism is very complex, and hyperglycemia, insulin resistance and hyperinsulinemia, obesity and chronic inflammation may play an important role in this process. Patients with diabetes are at high risk of cancer. Therefore, patients with diabetes should be actively screened during clinical diagnosis and treatment, such as detection of tumor markers in serum, so as to detect tumors as early as possible.

In conclusion, the levels of tumor markers CA199, CEA, and CA125 in patients with T2DM are significantly higher than those in the normal population. Smoking, family history of diabetes, hypertension, hyperlipemia, course of disease, CA199, CEA, CA153, CA125, AFP, SBP, DBP, FPG, HbA1c, M-ALB, and TG are the influential factors leading to complications in T2DM patients. Relevant indicators of T2DM patients should be fully evaluated clinically, and long-term follow-up observation should be conducted to reduce the incidence of complications.

\section{Acknowledgments}

Funding: The study was supported by Youth Science and Technology Fund of China University of Mining and Technology (No. 2020QN81).

\section{Footnote}

Reporting Checklist: The authors have completed the STROBE reporting checklist. Available at https://apm. amegroups.com/article/view/10.21037/apm-21-3429/rc

Data Sharing Statement: Available at https://apm.amegroups. com/article/view/10.21037/apm-21-3429/dss

Conflicts of Interest: All authors have completed the ICMJE uniform disclosure form (available at https://apm. amegroups.com/article/view/10.21037/apm-21-3429/coif). The authors have no conflicts of interest to declare.

Ethical Statement: The authors are accountable for all aspects of the work in ensuring that questions related to the accuracy or integrity of any part of the work are appropriately investigated and resolved. All procedures performed in this study involving human participants were in accordance with the Declaration of Helsinki (as revised in 2013). The study was approved by the ethics committee of The Affiliated Hospital of China University of Mining and Technology (No. LLSL-2021-62) and informed consent 
was taken from all the patients.

Open Access Statement: This is an Open Access article distributed in accordance with the Creative Commons Attribution-NonCommercial-NoDerivs 4.0 International License (CC BY-NC-ND 4.0), which permits the noncommercial replication and distribution of the article with the strict proviso that no changes or edits are made and the original work is properly cited (including links to both the formal publication through the relevant DOI and the license). See: https://creativecommons.org/licenses/by-nc-nd/4.0/.

\section{References}

1. Saeedi P, Petersohn I, Salpea P, et al. Global and regional diabetes prevalence estimates for 2019 and projections for 2030 and 2045: Results from the International Diabetes Federation Diabetes Atlas, 9th edition. Diabetes Res Clin Pract 2019;157:107843.

2. Yan BH, Cai Y, Xue M, et al. Comparative analysis on amenable mortality with main non-communicable diseases in urban and rural areas in China, 2010 and 2017. Zhonghua Yu Fang Yi Xue Za Zhi 2020;54:679-84.

3. Wang L, Gao P, Zhang M, et al. Prevalence and Ethnic Pattern of Diabetes and Prediabetes in China in 2013. JAMA 2017;317:2515-23.

4. Donath MY, Shoelson SE. Type 2 diabetes as an inflammatory disease. Nat Rev Immunol 2011;11:98-107.

5. Fabiani S, Fallahi P, Ferrari SM, et al. Hepatitis $C$ virus infection and development of type 2 diabetes mellitus: Systematic review and meta-analysis of the literature. Rev Endocr Metab Disord 2018;19:405-20.

6. Tharek Z, Ramli AS, Whitford DL, et al. Relationship between self-efficacy, self-care behaviour and glycaemic control among patients with type 2 diabetes mellitus in the Malaysian primary care setting. BMC Fam Pract 2018;19:39.

7. Liu QR, Ma LL. The serum levels and clinical significance of adiponectin in endometrial cancer with type 2 diabetic patients. Acta Academiae Medicinae Weifang 2015;37:422-3.

8. Liska V, Treska V, Skalicky T, et al. Evaluation of Tumor Markers and Their Impact on Prognosis in Gallbladder, Bile Duct and Cholangiocellular Carcinomas - A Pilot Study. Anticancer Res 2017;37:2003-9.

9. Liao KF, Lai SW, Li CI, et al. Diabetes mellitus correlates with increased risk of pancreatic cancer: a populationbased cohort study in Taiwan. J Gastroenterol Hepatol
2012;27:709-13.

10. World Health Organization. Definition, diagnosis and classification of diabetes mellitus and its complications. Part1: diagnosis and classification of diabetes mellitus. Geneva: World Health Organization,1999.

11. Gata VA, Lisencu CI, Vlad CI, et al. Tumor infiltrating lymphocytes as a prognostic factor in malignant melanoma. Review of the literature. J BUON 2017;22:592-8.

12. Zhao J, Han X, Xie M, et al. Epidemiological analysis of patients with type 2 diabetes malignant tumors. Chinese Journal of Prevention and Control of Chronic Diseases 2018;26:514-6.

13. Yang JH, Zhang LY, Chang BC, et al. Glycolipid metabolism and islet $\beta$ cell function in type 2 diabetic patients with a history of malignant tumor. Chinese Journal of Diabetes Mellitus 2018;10:404-9.

14. Liu MX, Jin L, Sun SJ, et al. Metabolic reprogramming by PCK1 promotes TCA cataplerosis, oxidative stress and apoptosis in liver cancer cells and suppresses hepatocellular carcinoma. Oncogene 2018;37:1637-53.

15. Shang $X$, Song C, Du X, et al. The serum levels of tumor marker CA19-9, CEA, CA72-4, and NSE in type 2 diabetes without malignancy and the relations to the metabolic control. Saudi Med J 2017;38:204-8.

16. Huang YP, Wu L, Mao F, et al. Intervention effects of " $5+1$ " grading management mode of diabetes in communities on the screening of T2DM complications. Chinese Journal of Prevention and Control of Chronic Diseases 2019;27:247-51.

17. Idowu AA, Ajose AO, Adedeji AT, et al. Microalbuminuria, Other Markers of Nephropathy and Biochemical Derangementsin Type 2 Diabetes Mellitus: Relationships and Determinants. Ghana Med J 2017;51:56-63.

18. Rehman K, Fatima F, Akash MSH. Biochemical investigation of association of arsenic exposure with risk factors of diabetes mellitus in Pakistani population and its validation in animal model. Environ Monit Assess 2019;191:511.

19. Bai HH, Zhou Z, Deng N. Risk factors for vascular complications in patients with type 2 diabetes mellitus. Hainan Medical Journal 2018;29:1802-4.

20. Lontchi-Yimagou E, Sobngwi E, Matsha TE, et al. Diabetes mellitus and inflammation. Curr Diab Rep 2013;13:435-44.

21. Donath MY, Schumann DM, Faulenbach M, et al. Islet inflammation in type 2 diabetes: from metabolic stress to therapy. Diabetes Care 2008;31 Suppl 2:S161-4.

22. Böni-Schnetzler M, Thorne J, Parnaud G, et al. 
Increased interleukin (IL)-1beta messenger ribonucleic acid expression in beta -cells of individuals with type 2 diabetes and regulation of IL-1beta in human islets by glucose and autostimulation. J Clin Endocrinol Metab 2008;93:4065-74.

23. Wellen KE, Hotamisligil GS. Inflammation, stress, and diabetes. J Clin Invest 2005;115:1111-9.

24. Kim KN, Joo NS, Je SY, et al. Carcinoembryonic antigen level can be overestimated in metabolic syndrome. J Korean Med Sci 2011;26:759-64.

25. Zayed AA, Beano AM, Amer FN, et al. Serum levels of carcinoembryonic antigen in patients with type 2 diabetes. Endocr Pract 2016;22:1310-8.

26. Lu HX, Lu XH, Zhang X, et al. Analysis the influence on the level of the serum CA125 factor in type 2 diabetic nephropathy patients. Ningxia Medical Journal 2016;38:228-9.

27. Xie F, Xie L, Li X, et al. Prevalence and risk factors of hypertension combined with diabetes in middle and elder population in Nan'an district of Chongqing. Zhonghua Liu Xing Bing Xue Za Zhi 2019;40:666-9.

28. Miao ZF, Xu H, Xu YY, et al. Diabetes mellitus and the risk of gastric cancer: a meta-analysis of cohort studies. Oncotarget 2017;8:44881-92.

Cite this article as: Cai R, Kong Q, Wang Z, Gao Z, Huo Y. Correlation between tumor markers and type 2 diabetes mellitus complications and their related influencing factors. Ann Palliat Med 2022;11(1):58-67. doi: 10.21037/apm-21-3429
29. Fang H, Yao B, Yan Y, et al. Diabetes mellitus increases the risk of bladder cancer: an updated meta-analysis of observational studies. Diabetes Technol Ther 2013;15:914-22.

30. Guo L, Yu M, Zhong J, et al. Stroke Risk among Patients with Type 2 Diabetes Mellitus in Zhejiang: A PopulationBased Prospective Study in China. Int J Endocrinol 2016;2016:6380620.

31. Wang L, Wang L, Zhang J, et al. Association between diabetes mellitus and subsequent ovarian cancer in women: A systematic review and meta-analysis of cohort studies. Medicine (Baltimore) 2017;96:e6396.

32. Andersen DK, Korc M, Petersen GM, et al. Diabetes, Pancreatogenic Diabetes, and Pancreatic Cancer. Diabetes 2017;66:1103-10.

(English Language Editor: C. Betlzar) 